\title{
Breathing Cycle and Posture Affect Magnitude and Anatomic Measurement Site of Waist Girth in Healthy Adults: An Insight from 3D Scanning
}

\author{
Clinton O. NJOKU ${ }^{1}$, Arthur D. STEWART ${ }^{2}$ \\ ${ }^{1}$ Department of Anatomy, Ebonyi State University, Abakaliki, Nigeria; \\ 2 School of Health Sciences, Robert Gordon University, Aberdeen, UK
}

DOI: $10.15221 / 18.190$ http://dx.doi.org/10.15221/18.190

\begin{abstract}
Waist girth is widely accepted as a simple anthropometric indicator of metabolic and cardiovascular disease risks. The aim of this research is to evaluate the impact of breathing cycle on the magnitude and anatomic measurement sites for waist girth using Hamamatsu 3D scanning. A sample of healthy adult volunteers ( 75 males and 36 females; age $27.8 \pm 7.5 y$ and $23.6 \pm 4.2 y$ respectively) participated in the study. Each wore form-fitting clothing (a swim cap, swimwear or lycra shorts and a sports top for women) which exposed the waist region. Each participant was scanned using a Hamamatsu BLS 9036 fixed scanner (Hamamatsu Photonics, UK) in three different phases of breathing cycle: end tidal $(\mathrm{T})$, inspired (I) and expired (E); and in a scanner posture (SP) with arms and legs abducted. Acquired scans were analysed using the system's software (Body Line Manager Version 1.3). The effect of the breathing cycle on waist girth had the highest mean value at $T(72.0$ and $83.9 \mathrm{~cm})$ in females and males, respectively and least mean value at $E(70.9$ for female and $81.9 \mathrm{~cm}$ for male). Adopting the scanner position resulted in a mean value of $70.5 \mathrm{~cm}$ and $82.9 \mathrm{~cm}$ for female and male respectively. At I, breathing cycle also altered waist girth significantly from the value obtained at end tidal $(P<0.05)$ in females but yielded no difference in males $(P>0.05)$. The anatomic measurement site for minimum waist girth had the highest vertical location at $E(115.0$ and $106.4 \mathrm{~cm})$ for male and female respectively, the least at I $(112.9$ and $105.0 \mathrm{~cm})$ for males and females respectively $(P<0.05)$. In the scanner position end tidal the height level was at $114.7 \mathrm{~cm}$ and $105.1 \mathrm{~cm}$ for males and females, respectively. Breathing cycle and posture affect measurement value and anatomic measurement site of waist girth.
\end{abstract}

\section{Introduction}

Waist girth (WG) is widely accepted as a simple anthropometric indicator of metabolic and cardiovascular disease risks. WG which typically represents a surrogate measure of the visceral fat area (VFA) [1,2] is always used as criteria for metabolic syndrome's diagnosis which are characterized by central obesity, impaired glucose tolerance, high blood pressure, and abnormal lipid metabolism [1]. Bigaard et al. [3] reported that waist girth remained strongly and directly associated with all-cause mortality when adjusted for total body fat in middle-aged men and women; suggesting that the increased mortality risk related to excess body fat is mainly due to abdominal adiposity.

Its magnitude is highly influenced by the anatomic measurement site $[4,5]$. Different groups of individuals adopt different postures with WG taken at different anatomic sites: ISAK recommends that WG be taken at the minimum part of the waist area in standing position with the measured holding the arms across the chest [6]; World Health Organization (WHO) [7] and Health Canada [8] recommend measurement at the midpoint between the superior border of the iliac crest and the lowest rib; US National Institutes of Health guidelines take above the superior border of the iliac crest as the appropriate site for WG measurement[9].

Accurate assessment of WG has important implications because a meta-regression analysis found that a $1 \mathrm{~cm}$ increase in WG increased the relative risk of cardiovascular disease by $2 \%$ [10]. A simple measurement error can lead to underestimation and mislead the measured to assume safe from risk for some conditions and diseases, just as an over estimation may result to unneeded anxiety on the subject.

In addition to waist location, the effects of posture and breathing on waist girth are unknown. Thus, the aim of this research is to evaluate the impact of breathing cycle on the magnitude and anatomic measurement sites for waist girth using Hamamatsu 3D scanning. 


\section{Methodology}

\subsection{Sample}

A sample of healthy adult volunteers (75 males and 36 females; age $27.8 \pm 7.5 y$ and $23.6 \pm 4.2 y$ respectively) recruited based on advertisement via a body composition flier and via e-mails seeking healthy adults for a single session body composition measurements. The participants were drawn from various ethnicities (Caucasians, Black Africans, Asians and Indians) but the greatest proportion came from a black African student community resident in Aberdeen and was mostly Nigerians. To ensure that the waist area is exposed, each participant wore form-fitting clothing (a swim cap, swimwear or lycra shorts and a sports top for women) which exposed the waist region. Owing to hormonal changes during pregnancy and the consequence on altered tissue masses, distribution and densities, pregnant women were excluded. The protocols for this study were approved by the Robert Gordon University, (RGU) Aberdeen Research Ethics Committee.

\subsection{Procedures for body scanning using the Hamamatsu Photonic 3DS}

The 3D scans were acquired using a Hamamatsu BLS 9036 fixed scanner (Hamamatsu Photonics, UK). Different scans were acquired by participants adopting different postures, and being at different phase of the breathing cycle. In the 'egress' position the participant stood upright with legs adducted to the midline and the arms extended and medially rotated to fix the palms on the lateral aspect of the thigh. For the 'scanner' position, the feet were shoulder width apart and legs and arms were abducted from the midline and with the hands extended, the palms were oriented in an anterioposterior axis. Following the instructions from the operator, three different scans were extracted in egress position: end tidal (T), inspired (I) and expired (E); while a posture: end tidal was scanned for scanner position (SP). The time for each measurement lasted for approximately 10 seconds (acquisition mode was set to high resolution). To ensure reproducibility of WG extraction, 12 participants' WG was tested through technical error of measurement (TEM) and relative technical error of measurement (RTEM).
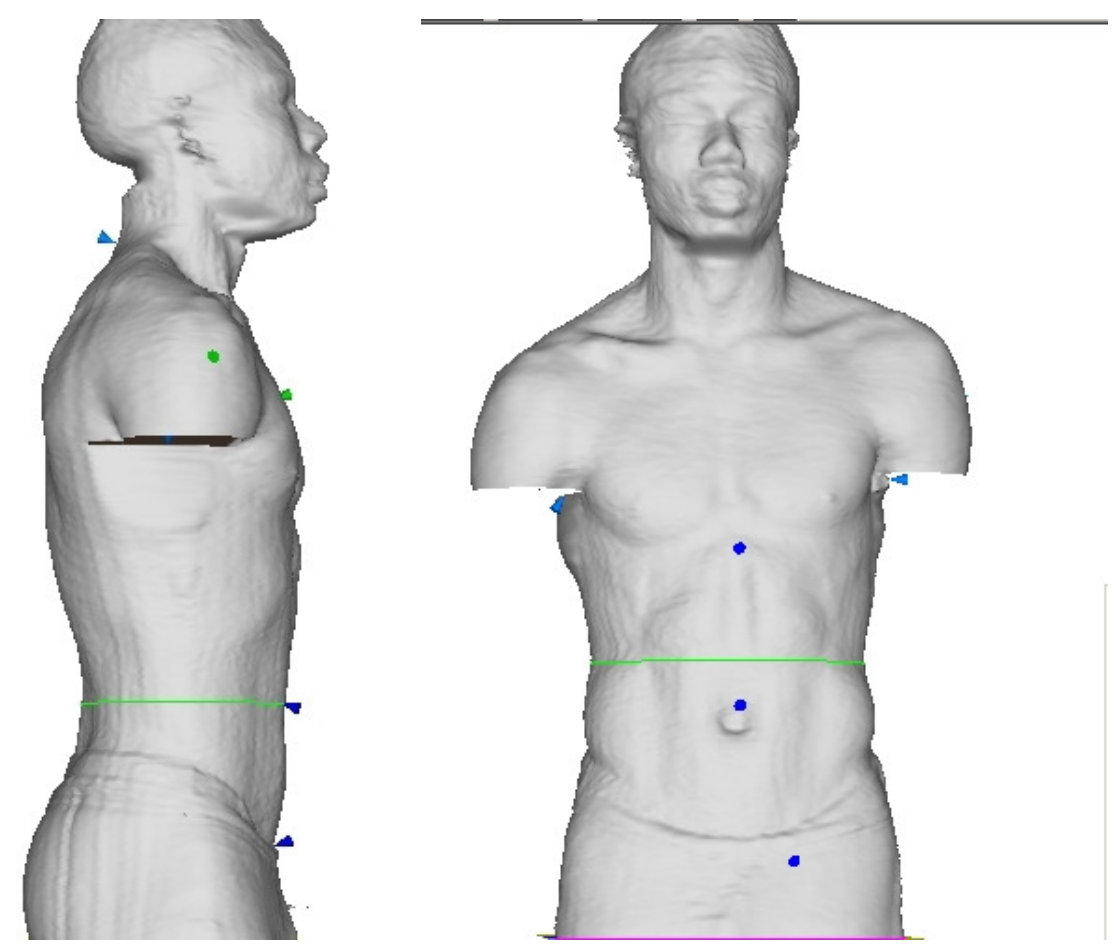

Figure 1. Front and side view of waist girth located at the minimum point of waist area as indicated with green line

\subsection{Scanner output and Data Extraction}

The scanner output involves a horizontal laser line array projected onto the body surface from four synchronized scan heads and merging the points acquired by different cameras as a point cloud. For the system's software (Body Line Manager version 1.3) to segment the body appropriately, five primary landmarks were identified at the vertex, C7 (nape), L and R axillae and crotch. All scans were 
processed and subsequently analysed using its proprietary software to produce a digital solid image that data can be extracted from. Through digital landmark placement (DLP), two digital landmarks were affixed on the solid digital image to identify the waist region (one on the lower border of the rib cage and the other was placed just above the iliac crest). The software then was automated to locate the point of minimum value of WG within the waist region (figure 1). Through this, the value of WG was acquired from the participants at different phases of breathing cycle in egress posture as well as at scanner posture.

\subsection{Statistical Analysis}

Before statistical analysis, the normality of the data was tested via a Kolmogorov-Smirnov test. Technical error of measurement (TEM) was used to evaluate measurement reproducibility. Descriptive statistics for all variables were expressed in mean \pm standard deviation. Paired T-tests were used to compare changes in WG during inspiration and expiration relative to end tidal, respectively. Statistical analysis was performed using SPSS version 21(SPSS Inc. Chicago, IL).

\section{Results}

\subsection{Impact of breathing cycle on waist girth measurements}

The reproducibility of waist girth was tested in 12 participants and TEM was $0.005 \mathrm{~cm}$. The relative TEM was $0.0057 \%$. The impact of the breathing cycle and scanner position (end tidal) on waist girth were analysed in 75 males and 36 females as presented in tables 1 and 2 . The effect of the breathing cycle on waist girth has the highest mean value at end tidal $(72.0$ and $83.9 \mathrm{~cm})$ in females and males, respectively and least mean value at egress position expired $(70.9$ for females and $81.9 \mathrm{~cm}$ for males). Adopting the scanner position resulted in a mean value of $70.5 \mathrm{~cm}$ and $82.9 \mathrm{~cm}$ for females and males, respectively. Breathing cycle altered waist girth significantly from the value obtained at end tidal $(P<0.05)$ except during inspiration in males where the waist girth extracted relative to that extracted at end tidal was not significant $(P>0.05)$.

Table 1. Paired-Sample Test analysis of waist girths according to breathing cycle and scanner position in females

\begin{tabular}{lccccc} 
& \multicolumn{7}{c}{$\mathbf{n = 3 6}$} & \\
& $\begin{array}{c}\text { Mean } \\
\text { diff }\end{array}$ & SD & \multicolumn{2}{c}{ 95\% Cl } & P-Value \\
& 1.0 & 1.9 & 0.3 & 1.5 & 0.04 \\
$\begin{array}{l}\text { WG egress end tidal and } \\
\text { WG egress inspired (cm) }\end{array}$ & & & & & \\
$\begin{array}{l}\text { WG egress end tidal WG } \\
\text { egress expired (cm) }\end{array}$ & 1.1 & 1.2 & 0.5 & 1.7 & 0.001 \\
$\begin{array}{l}\text { WG egress end tidal and } \\
\text { WG scanner end tidal (cm) }\end{array}$ & 0.9 & 1.0 & 0.5 & 1.4 & 0.001 \\
\hline
\end{tabular}

\section{WG = Waist Girth}

$P<0.05$ is significant

Table 2. Paired-Sample Test analysis of waist girth according to breathing cycle and scanner position in males

\begin{tabular}{|c|c|c|c|c|c|}
\hline \multicolumn{5}{|c|}{$n=75$} & \multirow{3}{*}{ P-value } \\
\hline & \multirow[t]{2}{*}{ Mean } & \multirow[t]{2}{*}{ SD } & \multicolumn{2}{|c|}{$95 \% \mathrm{Cl}$} & \\
\hline & & & Lower & Upper & \\
\hline $\begin{array}{l}\text { WG egress end tidal and } \\
\text { WG egress inspired }(\mathrm{cm})\end{array}$ & 0.4 & 2.4 & 0.3 & 1.1 & $>0.05$ \\
\hline $\begin{array}{l}\text { WG egress end tidal WG } \\
\text { egress expired }(\mathrm{cm})\end{array}$ & 2.0 & 1.8 & 0.3 & 1.5 & $<0.0001$ \\
\hline $\begin{array}{l}\text { WG egress end tidal } \\
\text { and WG scanner end } \\
\text { tidal }(\mathrm{cm})\end{array}$ & 1.5 & 3.1 & 0.4 & 1.3 & $<0.001$ \\
\hline
\end{tabular}




\subsection{Impact of breathing cycle on the extraction level of waist girth}

The height level above the standing surface at which waist girths were extracted was analyzed in the same participants as depicted on figure 2. For waist girth, egress position expired had the highest level $(115.0$ and $106.4 \mathrm{~cm})$ for males and females respectively, while in the egress inspired posture the height relative to egress end tidal was at $112.9 \mathrm{~cm}$ and $105.0 \mathrm{~cm}$ for males and females respectively. In the scanner position end tidal the height level was at $114.7 \mathrm{~cm}$ and $105.1 \mathrm{~cm}$ for males and females, respectively.

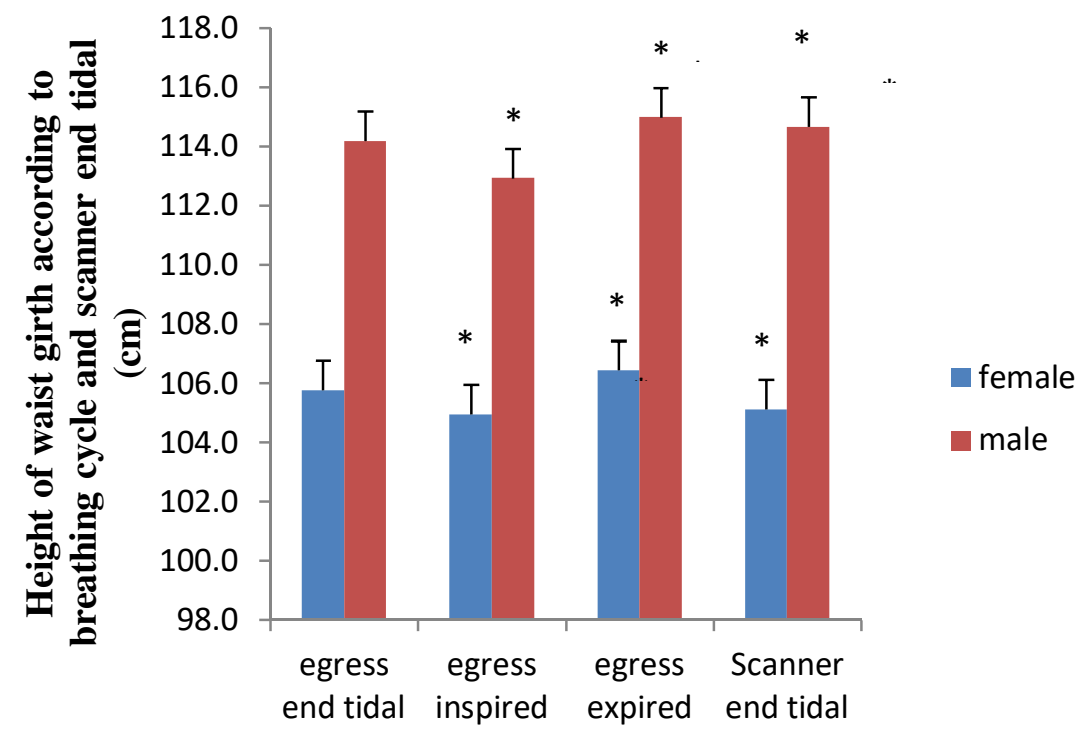

Breathing cycle and scanner end tidal

Figure 2. Bar charts showing mean height changes of waist girth according to breathing cycle.

Blue bars represent females and red represent males.

${ }^{*}$ Relative to the height of waist girth at egress end tidal $P<0.05$ in both sexes

\section{Discussion}

They are many factors that can lead to measurement errors of WG. Some of these are caused by the participants, the measurer and others from the equipment for WG extraction $[4,11,12,13,14,15$, 16].

Breathing cycle has impact both on the magnitude of $W G$ and also on the vertical height at which accurate measurements can be extracted. At the 'egress' position end tidal, waist girth has the highest mean values in both females and males, whereas at expiration the values for WG were reduced in females and males. This study also found that the level of WG extraction changed with breathing cycle with the WG at expiration having the highest vertical height from the standing surface of the participant. The height of WG extraction was least during inspiration.

This study's findings is similar to the findings of Agarwal et al[17] who reported that phases of respiration affect the magnitude of WG. The present finding has stood out because the posture at each breathing phase was acquired within $10 \mathrm{~s}$ and the narrowest part of the waist region was automated through proprietary software of the 3D scanner, unlike manual method that could consume more time with observer more likely to make mistakes regarding the narrowest point of the waist region. Breathing cycle also alters the level at which WG can be extracted. This supports the report of Mason and Kartmarzky [4] and Wang et al. [18], which reported that WG extracted from different anatomic sites vary significantly.

The concavity of females' waist region may be responsible for differences observed between $T$ and the other phases of breathing cycle, and explain why waist height appears less influential over waist magnitude in males. The anatomical distribution of fat cells (adipose tissues) is affected by the active sex hormones during and after puberty [19]. Just as circulating oestrogen stimulates fat cells to accumulate in gluteal region and thighs (gluteofemoral region) in women, and inhibits its accumulation in the abdominal region. On the other hand, circulating testosterone in men mainly stimulates accumulation of fat cells in the abdominal region and inhibits fat deposits in the gluteofemoral region. 
The present study had an excellent reproducibility. The TEM was $0.005 \mathrm{~cm}$ and relative TEM was $0.0057 \%$. However, our investigation was mainly on young adults with moderate body mass index $(\mathrm{BMI})$ and the minimal part of waist region was easily identified by 3D scanning. However, finding the minimal part of waist region on the obese whose waist regions are characterized by horizontal folds and furrows can pose difficulty for 3D scanning and may lead to inaccurate measurements. The obese also requires higher mechanical energy to enhance breathing.

\section{Conclusion}

Given the magnitude of these changes with breathing cycle, it may be surprising that protocols for 3D scanning have not previously specified breathing among their criteria. Therefore, to ensure best practice and accuracy, 3D scanning should be standardized for different postures and breathing phases.

\section{References}

1. Y. Matsuzawa, "Metabolic syndrome-definition and diagnostic criteria in Japan", In Japanese Society of Internal Medicine, 2005; 94:188-203.

2. S.M., Grundy, et al., "Diagnosis and management of the metabolic syndrome: an American Heart Association/National Heart, Lung, and Blood Institute Scientific Statement. American Heart Association.National Heart, Lung, and Blood Institute" Circulation", 2005; 112(17):2735-52.

3. J., Bigaard, et al., "Waist circumference and body composition in relation to all-cause mortality in middle-aged men and women", Int J Obes (Lond). 2005; 29(7):778-84

4. C., Mason, and P.T., Katzmarzyk, "Variability in waist circumference measurements according to anatomic measurement site", Obesity 17:1789 - 1795, 2009.

5. A.D., Stewart, et al., "Waist size and shape assessed by 3D photonic scanning", International Journal of Body Composition Research, 8(4) 123-130.

6. A.D., Stewart, et al., "International standards for anthropometric assessment. Lower Hutt, New Zealand" International Society for the Advancement of Kinanthropometry, 2011.

7. World Health Organization, "Obesity: preventing and managing Global Epidemic," Technical Report Series no. 894. Geneva: World Health Organization, 2000

8. Office of Nutrition Policy and Promotion, "Canadian Guidelines for body weight classification in adults". Health Canada: Ottawa <www.hc-sc.gc.cahpfbsaonpp_bwc_introduction_e.html> (2003).

9. "Clinical guidelines on the identification, evaluation, and treatment of overweight and obesity in adults: executive summary. Expert panel on the Identification, Evaluation, and Treatment of Overweight in Adults" American Journal of Clinical Nutrition, 1998; 68:899 - 917.

10. L., de Koning et al., "Waist circumference and waist-to-hip ratio as predictors of cardiovascular events: meta-regression analysis of prospective studies", European Heart Journal, 28:850-856, 2007.

11. T., Lohman et al., "Anthropometric standardization reference manual". Champagne, Illinois, Human Kinetic Books, 1988.

12. R., Gibson, "Principles of nutritional assessment". Oxford, Oxford University Press, 1990.

13. J.B., Croft et al., "Waist-to-hip ratio in a biracial population: measurement, implications, and cautions for using guidelines to define high risk for cardiovascular disease. Journal of the American Dietetic Association, 1995, 95(1):60-64.

14. Westat Inc., "National Health and Nutrition Examination Survey (NHANES) III". National Center for Health Statistics (NCHS), Centers for Disease Control and Prevention (CDC), 1998

15. WHO, "WHO STEPwise approach to surveillance (STEPS)". Geneva, World Health Organization (WHO), 2008.

16. Ross R, Berentzen T, Bradshaw AJ et al. Does the relationship between waist circumference, morbidity and mortality depend on measurement protocol for waist circumference? Obesity Reviews, 2008, 9(4):312-325.

17. S.K., Agarwal, et al., "Waist circumference measurement by site, posture, respiratory phase, and meal time: implications for methodology", Obesity (Silver Spring) 2009, 17, 1056-1061.

18. J., Wang et al., "Comparisons of waist circumferences measured at 4 sites", American Journal of Clinical Nutrition, 2003; 77:379-384.

19. A., Furnham et al. Waist-To-Hip Ratio and Preferences for Body Shape: A Replication and Extension Person. individ. DiJJ Vol. 22, No. 4, pp. 539-549, 1997 